\title{
Research on construction and evaluation of the reproduction color gamut for the future laser TV
}

\author{
Yan Li, Jing Wang and $\mathrm{Na} \mathrm{Li}$
}

\begin{abstract}
This article builds the 3D gamut with three primary colors and standard white light of the laser TV, compares the reproduction gamut of the laser TV and liquid crystal display, qualitatively analyses the difference of their gamut characteristic, and quantificationally calculates the 2D gamut coverage ratio and 3D gamut volume. The result shows that compared with 2D gamut, 3D gamut can be more comprehensive and objective for evaluating the monitor's color performance. This article also puts forward the realization rate of target gamut and gamut efficiency as the important parameters to evaluate the 3D reproduction ability of the displayer, thereby the objective evaluation system is created for the displayer. Using this system, this article evaluates the color effects of the future laser TV. It provides the theoretical values for ultra high-definition television system.
\end{abstract}

Keywords: Laser TV, Ultra high-definition television (UHDTV) system, 3D color gamut, Color gamut volume

\section{Introduction}

System of the recommendation ITU-R BT.709 [1] can only provide part of the visible colors that the human eyes can see. Because the reference RGB primaries defined in the recommendation were determined from the colorimetric characteristics of the phosphors generally adopted for a CRT monitor. So far, ITU-R BT.709 has being international recommendation for television broadcasting system whose gamut is called conventional gamut. But recent years, flat panel displays and cameras tend to be capable of reproducing and capturing more saturated colors located beyond the conventional gamut $[2,3]$.

In August 2012, the International Telecommunication Union (ITU) put forward the ultra-high-definition television (UHDTV) Recommendation ITU-R BT.2020 [4]. The realization of this recommendation can offer more realistic sensation, higher transparency to the real world, and more accurate visual information compared with HDTV system [5]. It realizes the wide-gamut display in the true sense. However, the three primary colors of UHDTV are located in the spectral locus. It is difficult to achieve its specified gamut for the existing liquid crystal display (LCD), plasma display panel displayers, so it is necessary to develop a kind of displayer to fulfill the

\footnotetext{
* Correspondence: wdxyliy@yahoo.com.cn

College of Physics and Electronic Information, Tianjin Normal University, Tianjin, China
}

recommendation UHDTV. Due to the good monochromaticity and high color purity of the laser [6], using the laser TV cannot only ensure the color of the reconstruction image, but also display the high saturation colors which are difficult to see in nature. In all, the future laser TV will be a development tendency to achieve the wide-gamut of UHDTV.

This article analyzes the reproduction gamut of the future laser TV with the constructed 3D gamut, and defines the performance parameters to evaluate the reproduction ability objectively. Finally, this article compares the gamut range between the future laser TV and the system of UHDTV/ITU-R BT.709.

\section{The comparative analysis of 2D chromaticity characteristic for the laser TV and LCD}

This article selects a laser TV [7] to compare with the traditional LCD. The chromaticity coordinates of three primary colors and standard white light for the two display equipments are shown in Table 1 , and the chromaticity diagram in CIE- $u^{\prime} v^{\prime}$ color space is illustrated in Figure 1.

As shown in Figure 1, the three primary colors' coordinates of laser TV are located on the spectrum locus. Its gamut that is the color scope fully contains the gamut of LCD. After transforming the chromaticity coordinates of the display device to CIE- $u^{\prime} v^{\prime}$ color space, 2D gamut coverage ratio [8] of laser is obtained. It is $59.43 \%$ far

\section{黑 Springer}


Table 1 Chromaticity coordinates of the laser TV/LCD

\begin{tabular}{ccccccccc}
\hline Coordinates & \multicolumn{3}{c}{ Laser } & \multicolumn{3}{c}{ LCD } \\
\hline Primary colors & $\boldsymbol{x}$ & $\boldsymbol{y}$ & $\boldsymbol{u}^{\prime}$ & $\boldsymbol{v}^{\prime}$ & $\boldsymbol{x}$ & $\boldsymbol{y}$ & $\boldsymbol{u}^{\prime}$ & $\boldsymbol{v}^{\prime}$ \\
Red & 0.713 & 0.287 & 0.5684 & 0.5147 & 0.6357 & 0.3412 & 0.4367 & 0.5273 \\
Green & 0.175 & 0.793 & 0.0575 & 0.5866 & 0.2795 & 0.6124 & 0.1142 & 0.5630 \\
Blue & 0.135 & 0.042 & 0.167 & 0.1169 & 0.1425 & 0.0572 & 0.1676 & 0.1514 \\
White & 0.3127 & 0.329 & 0.1978 & 0.4683 & 0.2726 & 0.2863 & 0.1851 & 0.4375 \\
\hline
\end{tabular}

higher than the color gamut coverage ratio of LCD's $33.5 \%$. That is to say that in $2 \mathrm{D}$ chrominance space without luminance factor, the color display effect of laser TV is better than the traditional LCD displayer.

\section{The comparative analysis of 3D chromaticity characteristic for laser TV and LCD}

\subsection{Establish 3D gamut of displayers}

CIE-LAB color space was established in 1976. It is more in line with the human visual requirements than others, and is considered as a uniform color space. So, 3D gamut of displayers are accomplished in CIE-LAB space, and the chromatic coordinates of colors are $L^{*}, a^{*}$, and $b^{*}$.

First of all, take the laser TV as an example to establish 3D gamut of laser TV in the CIE-LAB color space [9], which contains the luminance information and human eyes characteristic. According to the chromaticity coordinates of laser's three primary colors in Table 1, the relation

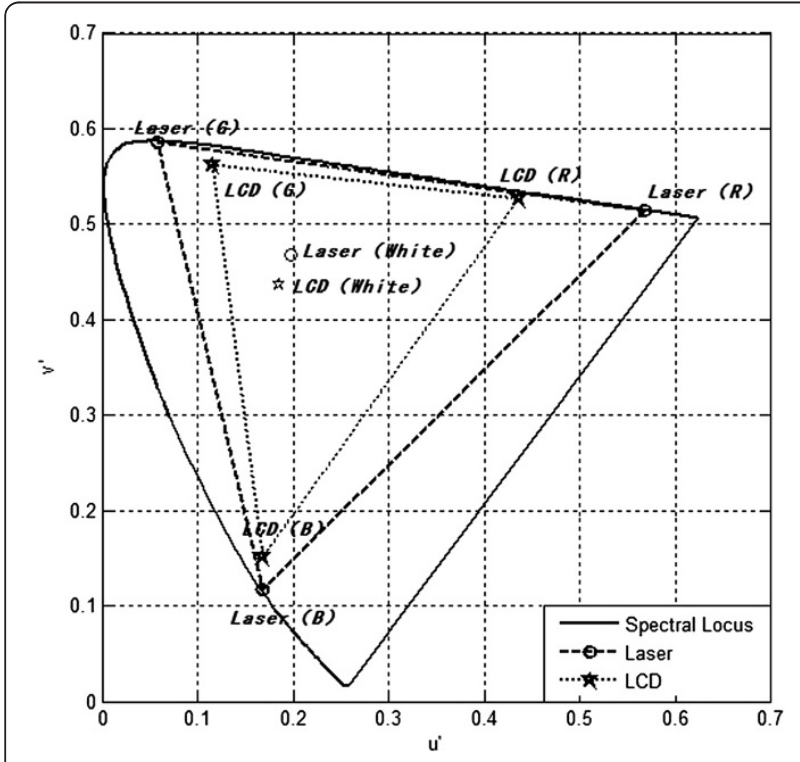

Figure $1 \mathrm{CIE}-u^{\prime} v^{\prime}$ chromaticity diagram of the laser TV/LCD. equation between the tristimulus values and unit amount of three primary colors [10] is found:

$$
\left[\begin{array}{ccc}
0.0623 & 0.1534 & 0.174 \\
0.2508 & 0.6951 & 0.0541 \\
0 & 0.028 & 1.0608
\end{array}\right]
$$

$\mathrm{Re}, \mathrm{Ge}, \mathrm{Be}$ is, respectively, the unit amount of the $\mathrm{R}$, G, B. Therefore, the brightness equation of laser TV is

$$
\mathrm{Y}=0.2508 \mathrm{Re}+0.6951 G e+0.0541 B e
$$

According to the brightness equation of laser TV and the principle of additive mixing color, the brightness of laser TV which belongs to $[0,1]$ can be divided into seven intervals, they are [0,0.0541], [0.0541,0.2508], [0.2508,0.3049], [0.3049,0.6951], [0.6951,0.7492], [0.7492,0.9459], and [0.9459,1], a total of eight brightness layers. Combined with formula 1, the gamut boundary points are found in this eight different brightness levels separately, and the tristimulus values of these points are transformed to the CIE-LAB color space. Thus the constructive 3D gamut of laser TV is shown as the part that envelope line surrounds in Figure 2.

\subsection{The comparative analysis of 3D chromaticity characteristic for laser TV and LCD displayer}

The 3D gamut of LCD is constructed with the method of 3-1 as the shadow part shown in Figure 2, comparative analysis the 3D display performance of laser TV and LCD.

Figure 2 is the three-dimensional chromaticity diagram of laser TV and LCD in the different angles of the CIE-LAB space. In Figure 2a,b, laser TV includes all the $3 \mathrm{D}$ color gamut that the $\mathrm{LCD}$ can show, but the LCD's gamut in a certain angle is beyond the scope of laser TV in Figure 2. The part out of the laser TV shows that the laser TV this article chooses is a little shorter than the LCD displayer in blue hue.

Laser TV contains all LCD's gamut in the 2D chromaticity diagram, but in 3D color space which adds the luminance information, laser TV is lightly worse than LCD in blue hue. So, it is necessary to establish the displayers' 3D gamut to analyze its display performance. 

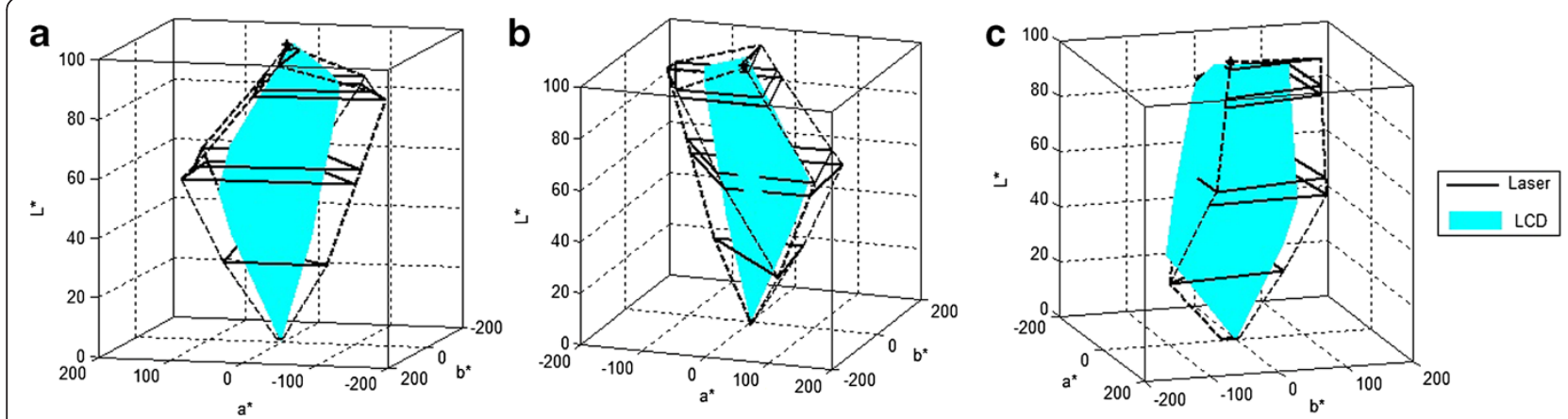

Figure 2 3D color gamut comparison diagram between laser TV and LCD.

3.3. The quantitative analysis for the size of 3D gamut According to Hill et al.'s theory [11], $\Delta L^{*}=\Delta a^{*}=\Delta b^{*}=1$ makes up the color cube in unit of one, the number of the small color cubes in the $3 \mathrm{D}$ gamut is the $3 \mathrm{D}$ gamut's volume.

First of all, the gamut boundaries are built with the incremental $\Delta L^{*}=1$ for the range of $L^{*}=[0,100]$ in the CIE-LAB color space. The gamut areas in which the gamut boundaries envelope in each layer are calculated, and therefore the 3D gamut size can be followed:

$$
V=\sum_{L^{*}=0}^{100} S_{L^{*}} \times \Delta L^{*}=\sum_{L^{*}=0}^{100} S_{L^{*}}
$$

where $V$ is the volume of the $3 \mathrm{D}$ gamut, $S_{L^{*}}$ is the area of the gamut boundary envelope in each layer, $\Delta L^{\prime \prime}$ is the distance between the each two layers. The results of formula 3 show that the size of LCD's 3D gamut is $8.93 \times 10^{5}$, while the size of laser TV's 3D gamut is $17.96 \times 10^{5}$ which is almost two times bigger than LCD's. So in the color display, the future laser TV is better than LCD display on the whole display effect.

Table 2 Chromaticity coordinates of laser TV/UHDTV/ITU-R BT.709

\begin{tabular}{|c|c|c|c|c|c|c|}
\hline & \multicolumn{2}{|c|}{ Laser } & \multicolumn{2}{|c|}{ ITU-R BT.709 } & \multicolumn{2}{|c|}{ UHDTV } \\
\hline & $x$ & $y$ & $x$ & $y$ & $x$ & $y$ \\
\hline Red & 0.713 & 0.287 & 0.64 & 0.33 & 0.7079 & 0.292 \\
\hline Green & 0.175 & 0.793 & 0.3 & 0.6 & 0.1702 & 0.7965 \\
\hline Blue & 0.135 & 0.042 & 0.15 & 0.06 & 0.1314 & 0.0459 \\
\hline \multirow[t]{2}{*}{ White } & 0.3127 & 0.329 & 0.3127 & 0.329 & 0.2726 & 0.2863 \\
\hline & $u^{\prime}$ & $v^{\prime}$ & $u^{\prime}$ & $v^{\prime}$ & $u^{\prime}$ & $v^{\prime}$ \\
\hline Red & 0.5684 & 0.5147 & 0.2134 & 0.5969 & 0.5565 & 0.5165 \\
\hline Green & 0.0575 & 0.5866 & 0.4324 & 0.4975 & 0.0557 & 0.5867 \\
\hline Blue & 0.1670 & 0.1169 & 0.1024 & 0.2956 & 0.1599 & 0.1256 \\
\hline White & 0.1978 & 0.4683 & 0.1978 & 0.4683 & 0.1978 & 0.4683 \\
\hline
\end{tabular}

The CIE- $u^{\prime} v^{\prime}$ diagram is drawn with the data in Table 2, the resultsare shown in Figure 3.

\section{Comparative analysis between laser TV and the system of UHDTV/ITU-R BT.709}

ITU-R BT.2020 sets the parameters values for UHDTV systems for production and international program exchange. UHDTV displays and broadcasting systems can offer more realistic sensation, accurate visual information compared with HDTV systems. This article analyzes the gamut character of the laser TV and recommendation of UHDTV/ITU-R BT.709, the parameters are listed in Table 2.

As shown in Figure 3, laser TV completely contains all the colors of the conventional gamut defined by ITU-R BT.709 in the CIE- $u^{\prime} v^{\prime}$ coordinate system, and its gamut coverage ratio out of ITU-R BT.709 is $15.93 \%$, and gamut coverage ratio out of UHDTV is $2.16 \%$, as shown in Table 3. From the chromaticity diagram, 2D gamut that is the new recommendation UHDTV specified is almost completely covered by the $2 \mathrm{D}$ gamut range of laser's. Therefore, laser TV as the future displayers can really realize wide-gamut display.

In Figure 4a, the part that the solid lines contain is the $3 \mathrm{D}$ gamut range of the laser $\mathrm{TV}$, while the part that the dotted lines envelope is the $3 \mathrm{D}$ gamut range of the recommendation UHDTV and the entity area in the center is the intersection of laser TV and UHDTV. The gamut of laser TV is fundamentally the same as UHDTV's. In Figure 4b, the part that the solid lines contain is also the 3D gamut range of the laser $\mathrm{TV}$, but the part enveloped by the dotted

Table 3 Comparison between laser TV and UHDTV/ITU-R BT.709

\begin{tabular}{cccc}
\hline & Laser & UHDTV & $\begin{array}{c}\text { ITU-R } \\
\text { BT.709 }\end{array}$ \\
\hline Color gamut area & 0.116 & 0.1118 & 0.0654 \\
$\begin{array}{c}\text { Color gamut coverage ratio } \\
\text { (\%) }\end{array}$ & 59.43 & 57.27 & 33.5 \\
Color gamut volume & $17.96 \times$ & $17.83 \times$ & $7.86 \times$ \\
& $10^{5}$ & $10^{5}$ & $10^{5}$ \\
\hline
\end{tabular}




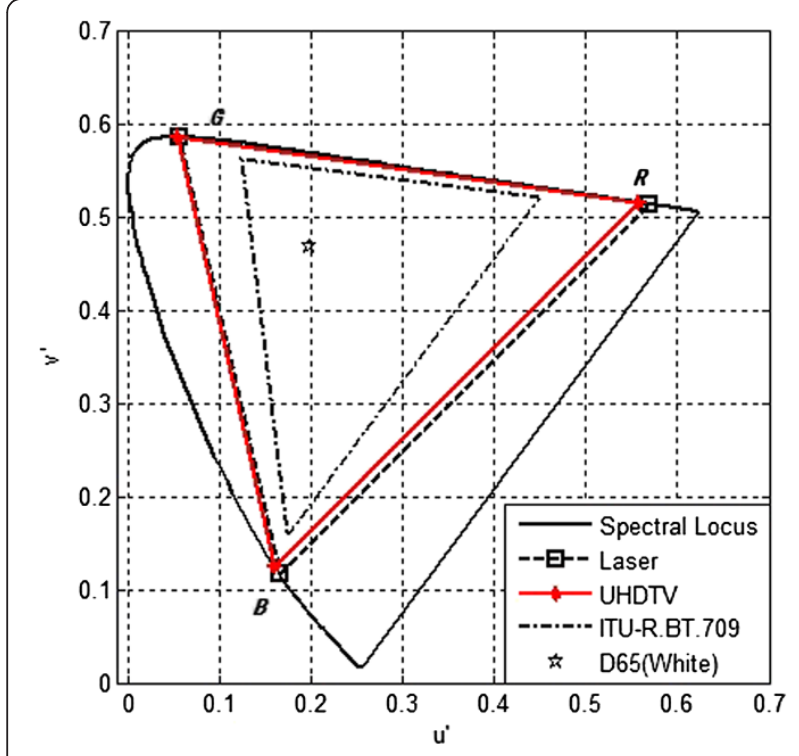

Figure $3 \mathrm{CIE}-u^{\prime} v^{\prime}$ gamut of the laser TV and UHDTV/ITU-R BT.709.

lines is the $3 \mathrm{D}$ gamut range of the system of ITU-R BT.709, and the entity in the center is the intersection of the gamut of laser TV and system of ITU-R BT.709. The gamut of laser TV contains the gamut of ITU-R BT.709 entirely. The result of quantitative calculation is shown in Table 3, the system gamut volume is $17.83 \times 10^{5}$ for recommendation UHDTV and $7.86 \times 10^{5}$ for ITU-R BT.709, while the laser TV's gamut volume is $17.96 \times 10^{5}$ which is big- ger than the values of recommendation UHDTV and ITU-R BT.709.

\section{Evaluation of the 3D gamut for laser TV}

Because of the TV standard's constraint, the displayer's gamut is not the bigger the better. It is much better to use smaller gamut to fully display the gamut which specified by the television standard as far as possible. This cannot only well display color gamut, but also reduce the hardware complexity and cost. Therefore, the realization rate of target gamut and the gamut efficiency $[12,13]$ are defined for the displayers as formulae (4) and (5) follow:

$$
\begin{aligned}
& \text { Realization rate of target gamut } \\
& =\frac{V_{\text {Equipment gamut }} \cap V_{\text {Target gamut }}}{V_{\text {Target gamut }}} \times 100 \%
\end{aligned}
$$

$$
\begin{aligned}
\text { Gamut efficiency }= & \frac{V_{\text {Equipment gamut }} \cap V_{\text {Target gamut }}}{V_{\text {Equipment gamut }}} \\
& \times 100 \%
\end{aligned}
$$

Equipment gamut is the gamut that the equipment can display, target gamut is the gamut which is specified by the television recommendations such as ITU-R BT.2020 and ITU-R BT.709. The realization rate of target gamut gives an indication that how fully the equipment gamut covers the target gamut. In order to get a good display effect, the intersection volume should be close to the target gamut volume so that the realization rate of target gamut is close to $100 \%$. The gamut efficiency means the percentage that the equipment gamut which is used to show the target gamut of the equipment, so higher gamut efficiency indicates that most of the equipment gamut is used to show the target gamut. If the gamut efficiency is much smaller, it means that the equipment gamut is bigger than the target gamut, and it needs to do the gamut compression. It will lead to color distortion.

The volume of the intersection part in 3D gamut, the realization rate of target gamut, and the gamut efficiency are calculated for the ITU-R BT.2020/laser and ITU-R BT.709/laser, respectively, and the results are shown in the following.
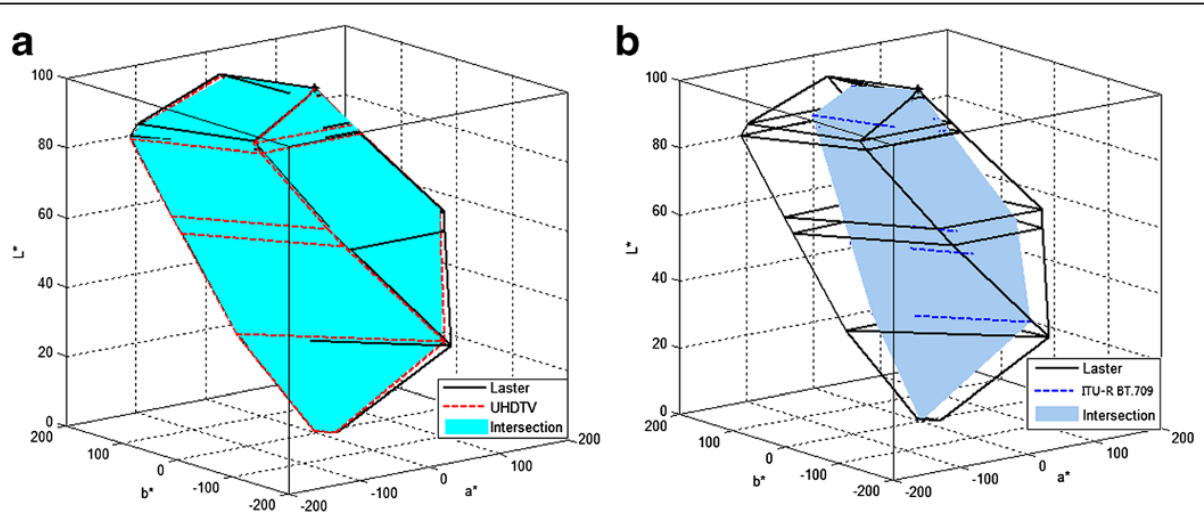

Figure 4 Intersection between laser TV and different standards. (a) Intersection between laser TV and UHDTV. (b) Intersection between laser TV and ITU-R BT.709 
Table 4 The 3D evaluation of the laser display

\begin{tabular}{ccc}
\hline & ITU-R BT.2020/laser & ITU-R BT.709/laser \\
\hline $\begin{array}{c}\text { Gamut intersection volume } \\
\text { Realization rate of target } \\
\text { gamut (\%) }\end{array}$ & $16.96 \times 10^{5}$ & $7.86 \times 10^{5}$ \\
Gamut efficiency (\%) & 95.12 & 100 \\
\hline
\end{tabular}

As showed in Table 4, the intersection volume between laser TV and the system of ITU-R BT.709 is $7.86 \times 10^{5}$, the realization rate of laser's target gamut is $100 \%$, but the gamut efficiency is only $43.76 \%$. It means that although the equipment gamut of laser TV is bigger than that of the system of ITU-R BT.709, but most of which is out of the gamut of the system ITU-R BT.709. The laser's gamut must be compressed to the scope of the recommendation ITU-R BT.709, the wide-gamut effect of laser TV cannot be achieved. This implies that the laser TV cannot keep a great compatibility with conventional gamut. However, for the new recommendation UHDTV, the intersection volume is $16.96 \times 10^{5}$, the realization rate of laser's target gamut is $95.12 \%$ and the value of the gamut efficiency is $94.43 \%$. The laser's gamut basically covered the gamut of ITU-R BT.2020 system, and higher gamut efficiency is reached. Therefore, laser TV will be a developing tendency for the new recommendation UHDTV in the future displayer market.

\section{Conclusion}

This article compares the reproduction ability of the laser TV and LCD in 2D chrominance space without the brightness information. The results show that laser TV fully contains the gamut that the LCD can display; but the same work in three-dimensional chromaticity diagram gets the different results that the gamut of LCD moves beyond the biggest gamut range of the laser TV in blue hue. In order to do the analysis in the 3D color space, this article constructs 3D color gamut for the laser TV.

In order to quantitatively analyze the size of the $3 \mathrm{D}$ gamut for laser TV, this article builds the laser TV's 3D gamut step-by-step in the CIE-LAB space, and uses the concept of color cube to calculate the gamut volume of laser TV. The volume is $17.96 \times 10^{5}$ which is two times bigger than LCD.

Furthermore, this article uses realization rate of target gamut and gamut efficiency to evaluate the laser TV's display performance. The laser TV can realize $100 \%$ of the recommendation ITU-R BT.709's gamut, but the gamut efficiency is only $43.76 \%$, which does not give full play to the wide-gamut of the laser TV. But for the UHDTV recommendation, laser TV cannot only better realize the gamut that the recommendation ITU-R BT.2020 specified, but also higher gamut efficiency is reached. So for, the future displayer market, the laser $\mathrm{TV}$, will be a good choice to realize the wide-gamut display under the UHDTV system.

\section{Competing interests}

I declare that we have no competing interests.

\section{Acknowledgment}

This study was supported by the Doctoral Foundation of Tianjin Normal University (52XB1006).

Received: 29 December 2012 Accepted: 7 March 2013

Published: 20 March 2013

\section{References}

1. International Telecommunication Union, ITU-R BT 7095:- Parameter Values for the HDTV Standards for Production and International Programme Exchange (ITU, Geneva, 2002)

2. H Kaneko, S Kagawa, J Someya, H Tanizoe, H Sugiura, Wide color gamut and high brightness WUXGA LCD monitor with color calibrators. IEICE Trans. Electron. E88-C, 2118-2123 (2005)

3. Y Shimodaira, H Suzuki, M Kretkowski, New imaging and display system for wide gamut color reproduction, in Conference Record of the2007 IEEE 42nd IAS Annual Meeting on Industry Applications Conference, 2007, pp. 1153-1157

4. International Telecommunication Union, ITU-R BT.2020: Parameter values for ultra-high definition television systems for production and international programme exchange (ITU, Geneva, 2012)

5. M Sugawara, K Masaoka, M Emoto, Y Matsuo, Y Nojiri, Research on human factors in ultrahigh-definition television (UHDTV) to determine its specifications. SMPTE Motion Imag. 117, 23-29 (2008)

6. G Song, Research on color reverting for display of new modes (Tianjin, Tianjin University, 2008)

7. K Moon-Cheol, Comparative color gamut analysis of XVYCC standard. Displays 4(29), 376-385 (2008)

8. Q Liu, Color gamut and color gamut coverage. Television Technol. 11, 49-52 (2004)

9. H Jiewei, T Shunqing, Z Zhengfang, Modern Color Science and Application (Beijing (Institute of Technology Press, Beijing, 2007)

10. L Sile, The Theory of Television (National Defence Industry Press, Beijing, 2007), pp. 37-39

11. B Hill, T Roger, FW Vorhagen, Comparative analysis of the quantization of color spaces on the basis of the CIELAB color difference formulate. ACM Transactions on Graphics 2(16), 109-154 (1997)

12. DS Park, SY Choi, HY Lee, YT Kim, JY Hong, CY Kim, A new wide-gamut RGB primary set and efficient color encoding methods for ultra high-definition television(UHDTV). Proceedings of the IEEE 101(1), 18-30 (2013)

13. G Braun, K Spaulding, Method for evaluating the color gamut and quantization characteristics of output-referred extended-gamut color encodings, in IS\&T/SID Tenth Color Imaging Conference (, New York, vol.10, November 2002), pp. 99-105

doi:10.1186/1687-1499-2013-80

Cite this article as: Li et al:: Research on construction and evaluation of the reproduction color gamut for the future laser TV. EURASIP Journal on Wireless Communications and Networking 2013 2013:80.

\section{Submit your manuscript to a SpringerOpen ${ }^{\circ}$ journal and benefit from:}

- Convenient online submission

- Rigorous peer review

- Immediate publication on acceptance

- Open access: articles freely available online

- High visibility within the field

- Retaining the copyright to your article

Submit your next manuscript at $>$ springeropen.com 